\title{
Un enfoque numérico en la demostración de un teorema del incremento del volumen de un cilindro de área mínima preservando su área
}

\section{A numerical approach in the proof of a theorem of increasing the volume of a cylinder of minimal area preserving its area}

\author{
Lizandro Baldomero Reyna Zegarra ${ }^{1}$, Heron Juan Morales Marchena ${ }^{1}$ y Santos López Rivera ${ }^{2}$ \\ 1 Departamento de Matemática, Universidad Nacional del Santa (UNS) \\ 2 Facultad de Ingeniería Agroindustrial, Universidad Nacional del Santa (UNS)
}

DOI: https://doi.org/10.33017/RevECIPeru2013.0011/

\section{Resumen}

Con la ayuda de técnicas numéricas, se propone una demostración de un teorema el cual establece que es posible incrementar el volumen de un cilindro de área mínima preservando su área.

Descriptores: preservación de áreas, incremento de volumen.

\begin{abstract}
With the help of numerical techniques, it is proposed a proof of a theorem which tells us that it is possible to increase the volume of a cylinder of minimal area preserving its area.
\end{abstract}

Keywords: preserving areas, increasing volume.

\section{Introducción}

Hace aproximadamente 15 años dos construcciones diferentes de curvamientos incrementando el volumen de un objeto geométrico han sido presentados [1,2]. En efecto, Bleecker [1], mostro que es posible comenzar con cualquier poliedro convexo y curvarlo a un poliedro no convexo de mayor volumen. En el 2006, Igor Pak [3] del departamento de matemáticas del Massachussets Institute of Technology (MIT), formulo un teorema que establece: "existe un poliedro no convexo cuya superficie es isométrica a la superficie de un cubo de menor volumen" cuya demostración no es complicada y se fundamenta en matemática elemental. En otras palabras, el teorema establece que es posible incrementar el volumen de un cubo preservando su área, dando como resultado final, la deformación del cubo al de un poliedro no convexo. Este poliedro no convexo tiene mayor volumen que el cubo, pero igual área. En el 2010, Lizandro Reyna y Heron Morales [4], plantearon un teorema análogo al planteado y demostrado por Pak, reemplazando el cubo por un cilindro de área mínima y el poliedro no convexo por un sólido de revolución del tipo no convexo.

El planteamiento de tal teorema surgió en base a resultados obtenido como consecuencia de la aplicación del Criterio de Optimización por Compensación [5], planteado con el fin de optimizar el diseño de botellas comerciales y lograr así ahorrar el uso de material en su fabricación.

La formulación del teorema análogo al planteado y demostrado por Pak, establece que: "existe un sólido de revolución del tipo no convexo cuya superficie 
Revista ECIPerú

Volumen 10, número 1

Septiembre 2013

es isométrica a la superficie de un cilindro circular recto de área mínima y de menor volumen que el de dicho solido de revolución", y cuya demostración analítica es requerida.

Aquí se plantea una demostración del teorema en la que necesariamente se precisa del uso de técnicas numéricas en una etapa de la demostración.

\section{Prueba del teorema}

En la figura 1 se plantea un esquema que en base al Criterio de Optimización por Compensación[5] y la condición de que la superficie del cilindro y la del solido de revolución sean isométricas[4], se obtengan relaciones matemáticas que permitan arribar a la demostración del teorema planteado.

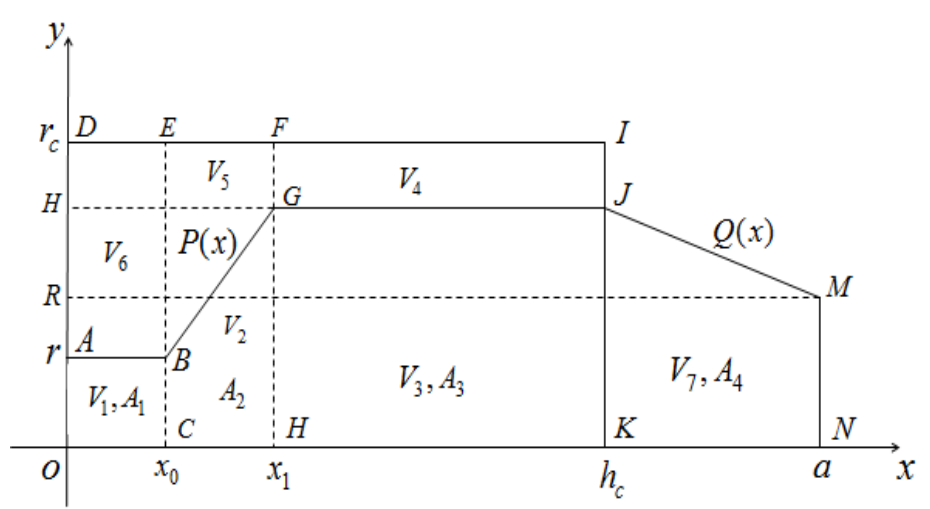

Figura 1

Del esquema de la figura 2 se tiene que:

$h_{c}$ y $r_{c}$ corresponden a la altura y radio de un cilindro de volumen $V_{c}$ y área mínima $A_{c}$ y que se genera al rotar el rectángulo ODIK alrededor del eje $\mathrm{x}$. Haciendo uso del cálculo diferencial se encuentra que $r_{c}=\frac{h_{c}}{2}$.

$P(x)$ y $Q(x)$ son polinomios enteros en $\mathrm{x}$ de primer grado cuyos coeficientes pueden ser determinados.

Los $A_{i}, i=0, \ldots, 5$, son las áreas generadas al rotar los respectivos segmentos $O A, A B, B G, G J, J M$ y $M N$ alrededor del eje $x$.

Los $V_{i}, i=1, \ldots, 7$, son los volúmenes generados al rotar las regiones limitadas por los polígonos cerrados respectivos $O A B C, C B G H, H G J K, G F I J, B E F G, A D E B$ y $K J M N$ alrededor del eje $\mathrm{x}$.

El valor $a$ es para ser determinado.
$H, r, R, x_{0}$ y $x_{1}$ son valores que se dan libremente con talque de que permitan determinar un valor para $a$ aceptable.

De la figura 1 se tiene que:

$V_{c}=V_{1}+V_{2}+V_{3}+V_{4}+V_{5}+V_{6}$

Además, por el Criterio de Optimización por Compensación,

$V_{7}=V_{4}+V_{5}+V_{6}$

Reemplazando (2) en (1)

$$
V_{c}=V_{1}+V_{2}+V_{3}+V_{7}
$$

También,

$$
\begin{aligned}
& V_{c}=\pi r_{c}^{2} h_{c} \\
& V_{1}=\pi r^{2} x_{0} \\
& V_{2}=\pi \int_{x_{0}}^{x_{1}}[P(x)]^{2} d x \\
& V_{3}=\pi H^{2}\left(h_{c}-x_{1}\right) \\
& V_{7}=\pi \int_{h_{c}}^{a}[Q(x)]^{2} d x \\
& P(x)=\alpha_{1} x+\alpha_{2} \\
& Q(x)=\beta_{1} x+\beta_{2}
\end{aligned}
$$

Reemplazando (9) en (6),

$$
\begin{aligned}
& V_{2}=\pi \int_{x_{0}}^{x_{1}}\left[\alpha_{1} x+\alpha_{2}\right]^{2} d x \\
& V_{2}=\pi \int_{x_{0}}^{x_{1}} \alpha_{1}^{2} x^{2}+2 \alpha_{1} \alpha_{2} x+\alpha_{2}^{2} d x
\end{aligned}
$$

Integrando,

$$
V_{2}=\pi\left[\alpha_{1}^{2} \frac{\left(x_{1-}^{3} x_{0}^{3}\right)}{3}+\alpha_{1} \alpha_{2}\left(x_{1}^{2}-x_{0}^{2}\right)+\alpha_{2}^{2}\left(x_{1}-x_{0}\right)\right]
$$

Luego,

$V_{2}=V_{2}\left(\alpha_{1}, \alpha_{2}\right)$

Reemplazando (10) en (8),

$$
\begin{aligned}
& V_{7}=\pi \int_{h_{c}}^{a}\left[\beta_{1} x+\beta_{2}\right]^{2} d x \\
& V_{7}=\pi \int_{h_{c}}^{a} \beta_{1}^{2} x^{2}+2 \beta_{1} \beta_{2} x+\beta_{2}^{2} d x
\end{aligned}
$$

Integrando, 
$V_{7}=\pi\left[\beta_{1}^{2} \frac{\left(a^{3}-h_{c}^{3}\right)}{3}+\beta_{1} \beta_{2}\left(a^{2}-h_{c}^{2}\right)+\beta_{2}^{2}\left(a-h_{c}\right)\right]$

Luego,

$V_{7}=V_{7}\left(\beta_{1}, \beta_{2}, a\right)$

Reemplazando (4), (5), (12), (7) y (14) en (3),

$\pi r_{c}^{2} h_{c}-\pi r^{2} x_{0}-V_{2}\left(\alpha_{1}, \alpha_{2}\right)-\pi H^{2}\left(h_{c}-x_{1}\right)-$

$V_{7}\left(\beta_{1}, \beta_{2}, a\right)=0$

Sea ahora,

$A_{c}=A_{0}+A_{1}+A_{2}+A_{3}+A_{4}+A_{5}$

donde

$A_{0}=\pi r^{2}$

$A_{1}=2 \pi r x_{0}$

$A_{2}=2 \pi \int_{x_{0}}^{x_{1}} P(x) \sqrt{1+\left[P^{\prime}(x)\right]^{2}} d x$

$A_{3}=2 \pi H\left(h_{c}-x_{1}\right)$

$A_{4}=2 \pi \int_{h_{c}}^{a} Q(x) \sqrt{1+\left[Q^{\prime}(x)\right]^{2}} d x$

$A_{5}=\pi R^{2}$

$A_{c}=2 \pi r_{c} h_{c}+2 \pi r_{c}^{2}$

Derivando (9),

$P^{\prime}(x)=\alpha_{1}$

Derivando (10),

$Q^{\prime}(x)=\beta_{1}$

Reemplazando (9) y (24) en (19) e integrando,

$A_{2}=2 \pi \sqrt{1+\alpha_{1}^{2}}\left[\alpha_{1} \frac{x_{1}^{2}}{2}+\alpha_{2} x_{1}-\alpha_{1} \frac{x_{0}^{2}}{2}-\alpha_{2} x_{0}\right]$

Luego,

$A_{2}=A_{2}\left(\alpha_{1}, \alpha_{2}\right)$

Reemplazando (10) y (25) en (21) e integrando,

$$
A_{4}=2 \pi \sqrt{1+\beta_{1}^{2}}\left[\beta_{1} \frac{a^{2}}{2}+\beta_{2} a-\beta_{1} \frac{h_{c}^{2}}{2}-\beta_{2} h_{c}\right]
$$

Luego,

$A_{4}=A_{4}\left(\beta_{1}, \beta_{2}\right)$

Reemplazando (17), (18), (27), (20), (29) y (22) en (16),
$2 \pi r_{c} h_{c}+2 \pi r_{c}^{2}-\pi r^{2}-2 \pi r x_{0}-A_{2}\left(\alpha_{1}, \alpha_{2}\right)-$ $2 \pi H\left(h_{c}-x_{1}\right)-A_{4}\left(\beta_{1}, \beta_{2}, a\right)-\pi R^{2}=0$

Condiciones que deben cumplir los polinomios $P(x)$ y $Q(x)$ :

$P\left(x_{0}\right)=r$

$P\left(x_{1}\right)=H$

$Q\left(h_{c}\right)=H$

$Q(a)=R$

De (31) y (9) se tiene que,

$\alpha_{1} x_{0}+\alpha_{2}-r=0$

De (32) y (9) se tiene que,

$\alpha_{1} x_{1}+\alpha_{2}-H=0$

De (33) y (10) se tiene que,

$\beta_{1} h_{c}+\beta_{2}-H=0$

De (34) y (10) se tiene que,

$\beta_{1} a+\beta_{2}-R=0$

Teniendo en cuenta (35), (36) , (37), (38), (15) y (30) se obtiene el siguiente sistema de ecuaciones,

$\alpha_{1} x_{0}+\alpha_{2}-r=0$

$\alpha_{1} x_{1}+\alpha_{2}-H=0$

$\beta_{1} h_{c}+\beta_{2}-H=0$

$\beta_{1} a+\beta_{2}-R=0$

$\pi r_{c}^{2} h_{c}-\pi r^{2} x_{0}-V_{2}\left(\alpha_{1}, \alpha_{2}\right)-\pi H^{2}\left(h_{c}-x_{1}\right)-$ $V_{7}\left(\beta_{1}, \beta_{2}, a\right)=0 \quad(43)$

$2 \pi r_{c} h_{c}+2 \pi r_{c}^{2}-\pi r^{2}-2 \pi r x_{0}-A_{2}\left(\alpha_{1}, \alpha_{2}\right)-$ $2 \pi H\left(h_{c}-x_{1}\right)-A_{4}\left(\beta_{1}, \beta_{2}, a\right)-\pi R^{2}=0$

Luego, se obtiene seis ecuaciones en cinco incógnitas.

De las ecuaciones (39) y (40) se obtiene

$\alpha_{1}=\frac{H-r}{x_{1}-x_{0}}$
$\alpha_{2}=\frac{r x_{1}-H x_{0}}{x_{1}-x_{0}}$

De las ecuaciones (41) y (42) se obtiene,

$\beta_{1}=\frac{R-H}{a-h_{c}}$ 
Reemplazando (11) y (13) en (43),

$$
\begin{gathered}
\pi r_{c}^{2} h_{c}-\pi r^{2} x_{0}-\pi\left[\alpha_{1}^{2} \frac{\left(x_{1}^{3}-x_{0}^{3}\right)}{3}+\alpha_{1} \alpha_{2}\left(x_{1}^{2}-x_{0}^{2}\right)+\right. \\
\left.\alpha_{2}^{2}\left(x_{1}-x_{0}\right)\right]-\pi H^{2}\left(h_{c}-x_{1}\right)-\pi\left[\beta_{1}^{2} \frac{\left(a^{3}-h_{c}^{3}\right)}{3}+\right. \\
\left.\beta_{1} \beta_{2}\left(a^{2}-h_{c}^{2}\right)+\beta_{2}^{2}\left(a-h_{c}\right)\right]=0
\end{gathered}
$$

Reemplazando (45), (46), (47) y (48) en (49) y simplificando,

$$
\begin{gathered}
\pi r_{c}^{2} h_{c}-\pi r^{2} x_{0}-\pi\left[\left(\frac{H-r}{x_{1}-x_{0}}\right)^{2} \frac{\left(x_{1}^{3}-x_{0}^{3}\right)}{3}+(H-r)\right. \\
\left.\left(r x_{1}-H r_{0}\right)\left(\frac{x_{1}+x_{0}}{x_{1}-x_{0}}\right)+\frac{\left(r x_{1}-H x_{0}\right)^{2}}{x_{1}-x_{0}}\right]-\pi H^{2} \\
\left(h_{c}-x_{1}\right)-\pi\left[\left(\frac{R-H}{a-h_{c}}\right)^{2} \frac{\left(a^{3}-h_{c}^{3}\right)}{3}+(R-H)\right. \\
\left.\left(H a-R h_{c}\right)\left(\frac{a+h_{c}}{a-h_{c}}\right)+\frac{\left(H a-R h_{c}\right)^{2}}{a-h_{c}}\right]=0
\end{gathered}
$$

La ecuación (50) es una ecuación en la variable $a$.

Reemplazando (26) y (28) en (44),

$$
\begin{aligned}
& 2 \pi r_{c} h_{c}+2 \pi r_{c}^{2}-\pi r^{2}-2 \pi r x_{0}-2 \pi \sqrt{1+\alpha_{1}^{2}}\left[\alpha_{1} \frac{x_{1}^{2}}{2}+\right. \\
& \left.\alpha_{2} x_{1}-\alpha_{1} \frac{x_{0}^{2}}{2}-\alpha_{2} x_{0}\right]-2 \pi H\left(h_{c}-x_{1}\right)-2 \pi \sqrt{1+\beta_{1}^{2}} . \\
& {\left[\beta_{1} \frac{a^{2}}{2}+\beta_{2} a-\beta_{1} \frac{h_{c}^{2}}{2}-\beta_{2} h_{c}\right]-\pi R^{2}=0}
\end{aligned}
$$

Reemplazando (45), (46), (47) y (48) en (51),

$$
\begin{aligned}
& 2 \pi r_{c} h_{c}+2 \pi r_{c}^{2}-\pi r^{2}-2 \pi r x_{0}-2 \pi \sqrt{1+\left(\frac{H-r}{x_{1}-x_{0}}\right)^{2}} . \\
& {\left[\left(\frac{H-r}{x_{1}-x_{0}}\right) \frac{x_{1}^{2}}{2}+\left(\frac{r x_{1}-H x_{0}}{x_{1}-x_{0}}\right) x_{1}-\left(\frac{H-r}{x_{1}-x_{0}}\right) \frac{x_{0}^{2}}{2}-\right.} \\
& \left.\left(\frac{r x_{1}-H x_{0}}{x_{1}-x_{0}}\right) x_{0}\right]-2 \pi H\left(h_{c}-x_{1}\right)-2 \pi \sqrt{1+\left(\frac{R-H}{a-h_{c}}\right)^{2}} \\
& {\left[\left(\frac{R-H}{a-h_{c}}\right) \frac{a^{2}}{2}+\left(\frac{H a-R h_{c}}{a-h_{c}}\right) a-\left(\frac{R-H}{a-h_{c}}\right) \frac{h_{c}^{2}}{2}-\right.} \\
& \left.\left(\frac{H a-R h_{c}}{a-h_{c}}\right) h_{c}\right]-\pi R^{2}=0
\end{aligned}
$$

La ecuación (52) al igual que la ecuación (50), es una ecuación en la variable $a$.

Resolviendo numéricamente la ecuación (50) para la variable $a$ con los datos de prueba dados libremente $r=1.2, r_{c}=4.43, h_{c}=8.86, x_{0}=2, H=4.2, R=2.7$ y $x_{1}=4.7$, se encuentra que $a=15.1384$. Teniendo en cuenta este último dato encontrado, el área y volumen del solido de revolución que se genera al rotar la región encerrada por la poligonal OABGJMN y el eje $x$ de la figura 1, alrededor del eje $x$, es igual a $360.684 \mathrm{~cm}^{2} y$ $546.249 \mathrm{~cm}^{3}$ respectivamente.

El área optima del cilindro de revolución que se genera al rotar la región encerrada por el rectángulo ODIK alrededor del eje $\mathrm{x}$, es igual a $369.921 \mathrm{~cm}^{2}$, mientras que su volumen es igual a $546.249 \mathrm{~cm}^{3}$ y que coincide con el valor del volumen del solido de revolución, razón por la cual se seleccionó la ecuación (50) para llevar a cabo la demostración.

Si designamos por $V_{S}$ y $A_{s}$ al volumen y área del solido de revolución, $V_{c}$ y $A_{c}$ al volumen y área del cilindro de área mínima, respectivamente, se tiene que $V_{s}=V_{c}$ y $A_{s}<A_{c}$. Lo que debemos ahora determinar es el volumen $V_{c}^{\prime}$ de un nuevo cilindro de área $A_{c}^{\prime}=A_{s}$ también mínima, de tal manera que se tenga $V_{c}^{\prime}<V_{s}$, con lo que el teorema quedaría demostrado.

En efecto, sabemos que el área de un cilindro cualquiera en función del radio $r$ está dado por

$A_{c}^{\prime}=2 \pi r^{2}+\frac{2 V_{c}^{\prime}}{r}$

donde $V_{c}^{\prime}$ es el volumen del cilindro.

Despejando $V_{c}^{\prime}$ de (53), se tiene,

$V_{c}^{\prime}=\frac{A_{c}^{\prime} r}{2}-\pi r^{3}$

Pero $A_{c}^{\prime}=360.684 \mathrm{~cm}^{2}$, luego de (54)

$V_{c}^{\prime}=180.342 r-\pi r^{3}$

Haciendo uso del cálculo diferencial para determinar los extremos de (55), se encuentra que el máximo ocurre en

$r=4.3743 \mathrm{~cm}$

y es único. 
Revista ECIPerú

Reemplazando (56) en (55), se encuentra que $V_{c}^{\prime}=$ $525.9186 \mathrm{~cm}^{3}$. Puesto que $V_{s}=546.249 \mathrm{~cm}^{3}$, se tiene en efecto que $V_{c}^{\prime}<V_{s}$ como se quería. El nuevo cilindro de volumen $V_{c}^{\prime}=525.9186 \mathrm{~cm}^{3}$ y área $A_{c}^{\prime}=$ $360.684 \mathrm{~cm}^{2}$, es un cilindro de área mínima, puesto que $V_{c}^{\prime}=525.9186 \mathrm{~cm}^{3}$ ha sido obtenido determinando el único máximo de (55) cuando $A_{c}^{\prime}=360.684 \mathrm{~cm}^{2}$. Esto último también se puede mostrar usando la fórmula para determinar el área mínima $A$ de un cilindro en función de su volumen $V$ y que se obtiene como resultado de determinar los extremos de (53). La fórmula está dada por,

$A(V)=6 \pi \sqrt[3]{\frac{V^{2}}{4 \pi^{2}}}$

Si remplazamos en (57) $V=V_{c}^{\prime}=525.9186 \mathrm{~cm}^{3}$, se obtiene $A=360.684 \mathrm{~cm}^{2}$ como se esperaba.

\section{Resultados y discusión}

Del esquema planteado en base al Criterio de Optimización por Compensación (Figura 1) se deducen dos ecuaciones (ecuaciones 50 y 52) en función de la variable $a$ que corresponde a la altura del solido de revolución del tipo no convexo. Las demás variables que aparecen en las referidas ecuaciones toman valores asignados libremente con tal de que de las ecuaciones se obtengan valores aceptables para $a$. Al ser resueltas estas ecuaciones haciendo uso de los métodos numéricos se encuentra que de la ecuación (50) para la diferencia de volúmenes y con los datos de entrada, se obtienen valores que son concordantes con el volumen y área mínima del cilindro. Luego, el área del solido de revolución de volumen igual a $546.249 \mathrm{~cm}^{3}$ resulto ser igual a $360.684 \mathrm{~cm}^{2}$ valor inferior al del cilindro de área mínima $369.921 \mathrm{~cm}^{2}$, en tanto que el volumen de un nuevo cilindro de área $360.684 \mathrm{~cm}^{2}$ resulto ser igual a $525.9168 \mathrm{~cm}^{3}$. Así se puede ver que las áreas se preservan pero que el volumen del solido de revolución de tipo no convexo es mayor que el volumen del nuevo cilindro de área mínima.

Idealmente se debería haber obtenido que las ecuaciones (50) y (52) dependan de dos variables con el fin de que la ecuación para la diferencia de volúmenes (ecuación 50) y la ecuación para diferencia de áreas (ecuación 52) estén presentes en la obtención de soluciones que contribuyan a la demostración del teorema. En principio para que esto sea así, se puede manipular haciendo que ambas
Septiembre 2013 ecuaciones dependan adicionalmente de una de las variables que se han manejado como de entrada, $H, r$, $R, x_{0}, 0 x_{1}$. Sin embargo, aun para el cálculo numérico se volverían tediosos los cálculos. Con suerte, la ecuación (50) proporciona el valor para la variable $a$, que es adecuado para lo que se necesita en la demostración del teorema materia del presente trabajo.

\section{Conclusiones}

Puesto que se ha encontrado que existe un cilindro de área mínima de volumen $V_{c}^{\prime}=525.9186 \mathrm{~cm}^{3}$ y área $A_{c}^{\prime}=360.684 \mathrm{~cm}^{2}$ así como un sólido de revolución de volumen $V_{s}=546.249 \mathrm{~cm}^{3}$ y área $A_{s}=360.684 \mathrm{~cm}^{2}$, se concluye que $A_{c}^{\prime}=A_{s}$ y $V_{c}^{\prime}<V_{s}$, que es lo que se quería demostrar.

Así, el teorema análogo al de Igor Pak y propuesto por Reyna - Morales queda demostrado. Las ilustraciones geométricas de ambos teoremas se muestran en las figuras 2 y 3 .

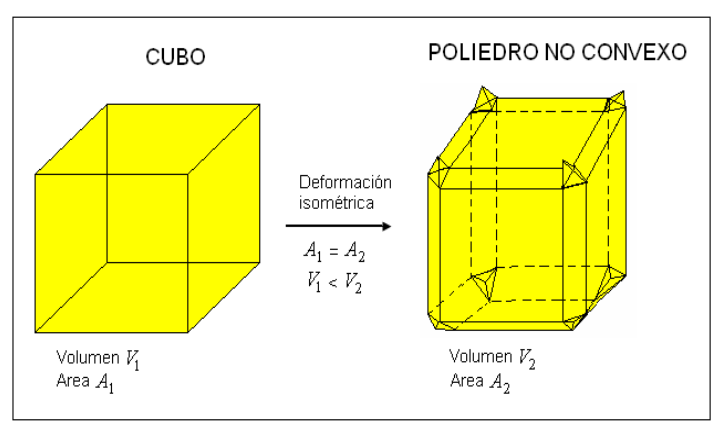

Figura 2: Deformación isométrica debido a Igor PaK

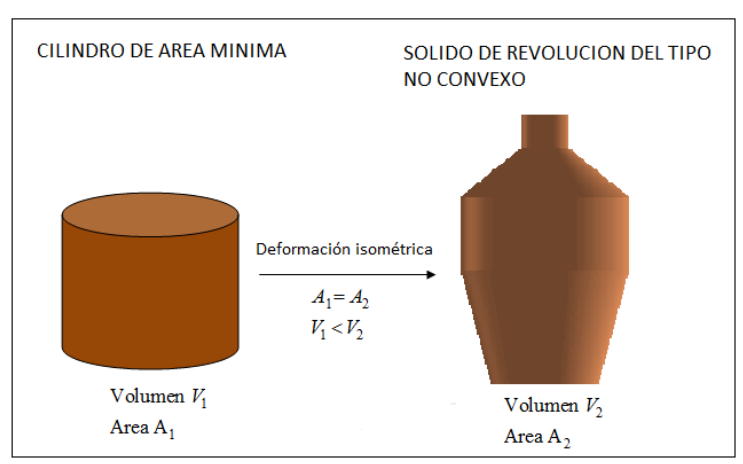

Figura 3: Deformación isométrica debido a Reyna Morales.

\section{Referencias}


[1] D. D. Bleecker. Volume increasing isometric deformations of convex polyhedral J. Diff. Geom. 43 (1996) 505-526.

[2] Yu. D. Burago and V. A. Zalgaller. Isometric piecewise - linear embeddings of two dimensional manifolds with a polyhedral metric into $R^{3}$ St. Petersburg Math. J. 7 (1996) 369385.

[3] Igor Pak. Inflating the cube without stretching, (2006) arXiv:math/0607754vl.
[4] Lizandro Reyna and Heron Morales. Isometric deformation of the surface of minimal area of a cylinder increasing its volume, Revista $\mathrm{ECl}, 7$ (2010) Número1.

[5] Lizandro Reyna y Teodoro Moore. Criterio de Optimización por Compensación en el diseño de botellas con superficies laterales de revolución usada por empresas en la comercialización de alimentos y bebidas. Revista ECl, 6 (2009) Número 1.

E-mail: reynazegarra@yahoo.es 